\title{
EFFICACY OF PROPOFOL AND MIDAZOLAM IN CONSCIOUS SEDATION FOR IMPLANT AND PERIODONTAL SURGERY
}

Amrita Gupta, Abhinav Gupta, Avanish Saxena, Parimal Sharma, Uma Srivastava, Yogita Dwivedi

1. Lecturer, Department of Anaesthesia, S.N. Medical College, Agra.

2. Assistant Professor, Department of Anaesthesia, Dr. Z.A Dental College, AMU Aligarh.

3. Associate Professor, Department of Anaesthesia, S.N. Medical College, Agra.

4. P. G. Student, Department of Anaesthesia. S. N. Medical College, Agra.

5. Professor, Department of Anaesthesia, S. N. Medical College, Agra.

6. Lecturer, Department of Anaesthesia, S.N. Medical College, Agra.

\section{CORRESPONDING AUTHOR}

Dr. Amrita Gupta,

89, Vimal Vatika, Agra,

Uttar Pradesh,

E-mail: amritagupta78@gmail.com,

Ph: 00919837077784.

ABSTRACT: A procedure which calms the patient with good control on psychomotor response will help the surgeons to perform surgery with perfection. One such procedure is the chair side sedation. OBJECTIVE: Evaluation of sedation technique which involved titrating intravenous midazolam to an ideal sedation end point, followed by continuous infusion of propofol. This technique might be satisfactory alternative to general anaesthesia for implant surgery in anxious patients or when procedure exceeds 60 mins duration. MATERIAL AND METHOD: The study was conducted on 20 patients. Initially $2 \mathrm{mg}$ bolus of midazolam was given then $1 \mathrm{mg}$ dose of the same drug was given every 30 seconds until the patients was adequately sedated. 10 minutes after the induction of sedation with midazolam a continuous infusion of propofol was started at the rate of 0-200 mg per hour. The quality of sedation was assessed using Ellis and Sedation scores. RESULT: Total midazolam dosage for adequate initial sedation of patient was between $6-14 \mathrm{mg}$ in 21 out of 23 treatment session. The initial propofol infusion rate was $200 \mathrm{mg}$ per hour in most of the cases. CONCLUSION: The sedation technique describe takes advantage of the differing pharmacokinetics properties of midazolam and propofol initial sedation was achieved using midazolam which was maintained throughout the surgeries variable propofol infusion .

KEYWORDS: Conscious sedation, midazolam, propofol

INTRODUCTION: In day to day oral and maxillofacial surgical outpatient procedures, the fear psychosis is unimaginable due to anxiety towards surgery and local anaesthesia administration. There will be considerable burden on time and expenditure on general anaesthesia which will increase morbidity and distress. It is highly unpleasant for the patients. Who is completely aware of the procedure.

Conscious sedation is used to depress the central nervous system in order to facilitate dental treatment; however unlike general anaesthesia the patient is able to respond to commands throughout the period of sedation. The main aim of the anaesthetist is to attain adequate level of sedation which enables dental treatment to be carried out safely with minimum level of distress to the patient. Short term recovery following intravenous sedation (within the first $60 \mathrm{~min}$ ) is related to distribution half life of the drug while full recovery depends on elimination half life. 
Benzodiazepines suitable for these procedures are diazepam and midazolam both drugs act by binding to receptor complex which facilitate GABA action (major inhibitory neurotransmitter in the brain). Midazolam is more potent and acts more rapidly than diazepam. Thus making it choice for producing amnesia during conscious sedation.

Midazolam is water soluble, non irritant to tissues. It has distribution half life of $6-30$ min and elimination half life of $1-4$ hour $^{1}$. Midazolam is metabolized in liver and its metabolite is inactive ${ }^{2}$.

Benzodiazepines can cause profound respiratory depression which is not physiologically compensated ${ }^{3}$. Therefore underlying principle for administering intravenous sedation with midazolam is to titrate incremental doses of drug according to the patients response. Signs of adequate anxiolysis and sedation include general relaxation and slurred speech $^{4}$.

Propofol is a sedative/hypnotic which also enhances GABA activity 5,6and induces depression of CNS so it can be used as an alternative to benzodiazepines for intravenous sedation 7 .Propofol has distribution half life of $2-4 \mathrm{~min}$ and elimination half life ranging from 30 - 60 min. Propofol undergoes hepatic metabolism into four inactive metabolite ${ }^{8}$.

Midazolam and Propofol are used synergistically because of distinct pharmacokinetic properties $9,10,11$. The use of midazolam in combination with Propofol has also been advocated for sedation ${ }^{12}$.

The present study explores the advantages of using a drug with very short distribution and elimination half life for maintenance of sedation. The sedation technique used involved titrating intravenous midazolam to an ideal sedation end point followed after 10 minutes by continuous infusion of Propofol, which maintained an optimum level of sedation and also resulted in prompt recovery. This study is an evaluation of existing sedation techniques in individuals undergoing routine dental treatment.

MATERIAL AND METHOD: The study was conducted on 20 healthy adult patients, 14 females and 6 males aged between 20-55 years who consented for conscious sedation. The surgery was done in the operation room, which was fully equipped with monitors including noninvasive blood pressure, heart rate, pulseoximetry, anaesthesia machine and emergency drugs .For emergency airway management kit for endotracheal intubation was available. Intravenous line was secured and each patient received glycopyrrolate $0.2 \mathrm{mg}$. For sedation $1 \mathrm{mg}$ bolus of midazolam was given and $0.5 \mathrm{mg}$ incremental doses of the same drug was repeated every 30 seconds till target sedation score of 3(eye closed, respond promptly on verbal command) achieved followed by continuous infusion of propofol starting at $200 \mathrm{mg}$ per hour using a syringe pump. When the target sedation score(3)was achieved the surgeon was asked to give local block. Routinely the inferior dental, infraorbital and posterior maxillary nerve blocks were used according to the area being treated. If the patient moved bolus of $0.5 \mathrm{mg}$ midazolam was given .All patients received lignocaine 2\%with 1:200000 epinephrine. Maximum safe dose of lignocaine with adrenaline is $5-7 \mathrm{mg} / \mathrm{kg}$.. Throughout the surgery the target sedation was achieved by regulating the propofol infusion rate. Blood pressure and pulse rate were monitored at 5 minutes interval throughout the surgery and SPO2 was monitored continuously. Oxygen was not given routinely but if SPO2 decreased below $90 \%$ for more than 60 seconds then oxygen via nasal mask was given. Each patient received $250-500 \mathrm{ml}$ of ringer lactate during the surgery. Intermittent suction was done throughout the surgery. Post operatively the pain response, and any type of discomfort due to procedure was noted. All patients were given 
antibiotics and analgesics postoperatively. Patients were reviewed every 48 hrs till the day of suture removal.

The depth of sedation (Table 1) and behavioural characteristics under sedation (Table 2) were assessed using the Ellis ${ }^{13}$ and sedation ${ }^{14}$ scoring technique. An Ellis score of I and sedation score of 3 were the target levels. The Propofol infusion was stopped at the completion of surgery.

Sedation scoring system is given in table 1 and Ellis behavioural scoring system is given in table 2

Table 1: The sedation scoring system used to grade the depth of sedation of patients under intravenous sedation.

1. Fully awake and oriented.

2. Drowsy

3. Eyes closed, responds promptly on verbal command.

4. Eyes closed, rousable on mild physical stimulation.

5. Eyes closed, unrousable on mild physical stimulation.

Table 2:- The Ellis scoring system used to grade the behavioural characteristics of patients under intravenous sedation.

I. No uninvited limb movement; total co-operation and no restlessness.

II. Small amount of uninvited limb movement; still total cooperation and no restlessness

III. More uninvited limb movement; small degree of restlessness and anxiety. Patient less co-operative; still able to perform all dental procedures.

IV. Considerable degree of limb movement; perhaps also unhelpful head movements; co-operation poor; patient quite restless and anxious; able to perform only basic dentistry; advanced, delicate work not possible.

V. Restlessness, anxiety and limb movements severe; impossible to perform any dentistry.

Any post operative complication like nausea, vomiting headache was recorded. The vitals such as heart rate blood pressure, oxygen saturation, respiratory rate were recorded 2 min before sedation, after the onset of sedation, 2 min after LA administration and followed by every 10 minutes interval of time i.e. $10^{\text {th }}, 20^{\text {th }}, 30^{\text {th }}$ and $40^{\text {th }}$ till the completion of surgery.

RESULTS: The 23 treatment sessions in the current investigations lasted from 60 to $150 \mathrm{~min}$ depending on the number of implant and nature of surgery. Total midazolam dosage fen adequate initial sedation of patients was between 6-14 mg with sedation scare of 3 (table 1) in 21 out of 23 treatment sessions. The initial Propofol infusion rate was $200 \mathrm{mg} / \mathrm{hr}(20 \mathrm{ml} / \mathrm{hr})$. This was adjusted throughout the surgery to maintain the sedation score of 3 in 21 out of 23 treatment sessions. Sedation score of 2 (drowsy) and 4 (eyes closed but reusable on mild physical stimulation) respectively were recorded in the remaining 2 patients. The total dose of Propofol administer to the patient was between $50 \mathrm{mg}$ to $350 \mathrm{mg}$. Throughout the surgery the arterial oxygen saturation was between $92-100 \%$ (without oxygen). All patients were fit to 
discharge within 4 hours of termination of Propofol infusion. No postoperative nausea, vomiting and headache were recorded in any patients.

DISCUSSION: Anxiety and fear are inevitable circumstances met by the patients undergoing oral surgical procedures. Conscious sedation in very helpful to carryout minor oral surgical procedures. Midazolam produced 30 - 40 min of profound amnesia, anxiolysis and sedation ${ }^{15}$. Amnesia was useful in reducing the memory of administration of LA and of oral surgery which are often perceived as unpleasant by an anxious patient. The total midazolam dosage required for adequate initial sedation was $6-10 \mathrm{mg}$. Like our study, the variability and unpredictability of the midazolam dose required to produce sedation in patients has been previously reported ${ }^{\mathbf{1 6}, \mathbf{1 7}}$. Factors such as anxiety, concurrent drug treatment, amount of sleep the night before operation and the level of stress encountered at work or in home are extremely variable and may explain why it is almost impossible to predict how much midazolam will be required for a particular patient ${ }^{18}$. There was tenfold variability between individuals liver content and catalytic activity of major liver enzyme involved in the metabolism of midazolam ${ }^{19,20,21}$ which could account for unpredictability of patient response for midazolam.

An initial Propofol infusion rate of $200 \mathrm{mg} / \mathrm{hr}(20 \mathrm{ml} / \mathrm{hr})$ was employed for 21 of the 23treatment session. Sedation score of 3 was maintained throughout the procedure satisfactorily by this dose. When propofol was used as sole agent the dose used was $300 \mathrm{mg}$ per hour. There is decrease in dose requirement of propofol when used in combination with midazolam is widely reported $11,22,23$ and it is because of fast acting nature of midazolam and the synergistic interactions of Propofol and midazolam ${ }^{24}$. While the use of midazolam in combination with Propofol was previously used for sedation ${ }^{12}$, these authors used midazolam as premedication prior to a bolus dose of Propofol, followed by Propofol infusion. There is unpredictability of midazolam ${ }^{\mathbf{1 6 , 1 7}}$ and Propofol ${ }^{25,7}$ when administered as bolus injections which increase the potential for both over sedation or under sedation. Our sedation technique involved titrating intravenous midazolam to an ideal sedation end point according to patient's response in order to avoid over sedation or under sedation. Taylor et al (1992) attempted to maintain a sedation score of 3 throughout the procedure ${ }^{12}$ but fixed initial propofol infusion rate did not allow operator to adjust the sedation level. 10 minutes after the administration of midazolam 1 patient was restless (Ellis score $=3$ ) in which the initial propofol infusion rate was increased to $300 \mathrm{mg}$ per hour and it was reduced after 30 minutes when the patient became more relaxed.The second patient had sedation score of 4 and Ellis score of 2 after 10 minutes of intravenous midazolam and in this case the initial propofol infusion was reduced to $50 \mathrm{mg}$ per hour. Infusion rate was increased to $150 \mathrm{mg}$ per hour when the sedation score was achieved to 3.

In the current investigation, the Propofol infusion rate was adjusted between 0 $\mathrm{mg} / \mathrm{hr}(0 \mathrm{ml} / \mathrm{hr})$ and $200 \mathrm{mg} / \mathrm{hr}(20 \mathrm{ml} / \mathrm{hr})$ depending on the level of sedation achieved with the titrated midazolam. By titrating midazolam to the patients needs and then varying the rate of infusion of a drug with short distribution and elimination life's the level of sedation was optimised throughout the surgical procedure. Using the sedation and Ellis scores it was shown that the quality of sedation was satisfactory for both the patient end the operator. None of the patient had significant respiratory compromise, hypotension, bradycardia, nausea, vomiting.

CONCLUSION: The sedation technique involved giving initial sedation with midazolam, this was then maintained using a variable propofol infusion. The shorter distribution and elimination 
half life's of the latter drug made it easier for the operator to match the level of sedation according to patient's need and also provided good short term recovery.

\section{REFERENCES:}

1. Wood, M. (1990) Intravenous anaesthetic agents. In: Drugs and anaesthesia pharmacology and anaesthesiologists, $2^{\text {nd }}$ edition, eds. Wood M. \& wood A.J.J., pp. 179 223. Baltimore: Williams and Wilkins.

2. Blumer J. I. (1998) Clinical pharmacology of midazolam in infants and children. Clinical Pharmacokinetics 35, $37-47$.

3. Coulthard, P. \& Craig, D. (1998) Conscious sedaion. Dental Update 24, 376-381.

4. Skelly, Am. (1992) Sedation in dental practice. Dental Update 19, 61 - 67.

5. Hales, T.G. \& Lambert, J.J. (1991) The actions of Propofol on inhibitory amino acid receptors of bovine adrenomedullary chromaffin cells and rodent central neurones. British Journal of pharmacology 104, $619-628$.

6. Hara, M., Kai, Y. \& Ikemoto, Y. (1994) Enhancement by Propofol of the y-aminobutyric acid response in dissociated hippocampal pyramidal neurons of the rat. Anesthesiology 81, 988-994.

7. Cillo, J. E. (1999) Propofol anesthesia for outpatient oral and maxillofacial surgery. Oral surgery Oral Medicine Oral Pathology Oral Radiology and Endodonitics 87, 530-538.

8. Schnider,T.W., Minto, c.F., gambus, P.L., Andresen, C., Goodale, D.B., Shafer, S. L. \& Youngs, E.J. (1998) The influence of method of administration and covariates on the pharmacokinetics of Propofol in adult volunteers. Anaesthesiology 88, 1170 - 1182.

9. Oxorn, D.C.,Ferris, L.E., Harrington, E. \& Orser, B.A. (1997) The effects of midazolam on Propofol induced anaesthesia Propofol dose requirements, mood profiles, and perioperative dreams. Anaesthesia Analgesia 85, 553 - 559.

10. Anderson, L. \& robb, H. (1998) A comparison of midazolam co-induction with Propofol predosing for induction of anaesthesia. Anaesthesia 53, $1117-1120$.

11. Carrasco et al. (1998) Synergistic sendation with Propofol and midazolam in intensive care patients after coronary artery bypass grafting critical medicine 26, 844851.

12. Taylor, E., Ghouri, A.F. \& White, P.F. (1992) Midazolam in combination with Propofol for sedation during local anaesthesia journal of Clinical anaesthesia 4,213-216.

13. Ellis, S. (1996) Response to intravenous midazolam sedation in general dental practice. British Dental Journal 180, 417-420.

14. Oei-Lim, V.L.B., Kalkman, C.J., makes, P.C., Ooms, W.G. \& Hoogstraten, J. (1997) Computer controlled infusion of Propofol for conscious sedation in dental treatment. British Dental Journal 183, $204-208$.

15. DC.Craig et al :A Sedation technique for implant and periodontal surgery.J Clin Periodontal 2000 semicolon 27:955-959

16. Dundee, j. W., Samuel, I. O., Toner, W. \& Howard, P.J. (1980) Midazolam: a water soluble benzodiazepine. Studies in volunteers. Anaesthesia 35, 454-458.

17. Richards, A., Griffiths, M. \& Scully, C. (1993) Wide variation in patient response to midazolam sedation for outpatient response to midazolam sedation for outpatient oral surgery Oral Medicine Oral Pathology 76, 408 - 411.

18. Girdler, N.M. \& Hill, C.M. (1998) Sedation in dentistry, pp. 73-96. Oxford: Butter-worthHeinemann. 
19. Lown, K.S., Thummel, K.E., Benedict, P.e., Shen, D.D., Turgeon, D.K., Berent, S. \& Watkins, P.B. (1995) The erythromycin breath test predicts the clearance of midazolam. Clinical pharmacology and Therapentics 57, $16-24$.

20. Kronbach, T., mathys, D., Umeno, M., Gonzalez, F.J. \& Meyer, U.A. (1989) Oxidation of midazolam and triazolam by human liver cytochrome P450IIA4. Molecular pharmacology 36, 89 - 96.

21. Bork R. W., Muto T.,Beaune P.H., Srivastava P.K.l Lloyd R.s. \& guengerich F.P. (1989) characterisation of mRNA species related to human liver cytochrome P-450 nifedipine oxidase and the regulation of catalytic activity. Journal of Biological Chemistry 264, 910 $-119$.

22. Tighe, K.E. \& Warner, J.A. (1997) The effect of co-indication with midazolam upon recovery from Propofol infusion anaesthesia. Anaesthesia 52, 998 - 1004.

23. Djaiani, G. \& Ribes-Pastor, M.P. (1999) Propofol anto-co-induction as an alternative to midazolam co-induction for ambulatory surgery. Anaesthesia 54, 63-67

24. McClune, S., Mckay, A.C., Wright, P.M.c., patterson, C.c. \& Clarke, R.S. (1992) Synergistic interaction between midazolam and Propofol. British Journal of Anaesthesia 69, 240 245.

25. MacKenzie, n. \& Grant, I.S. (1987)Propofol for intravenous sedation. Anaesthesia 42, 36.

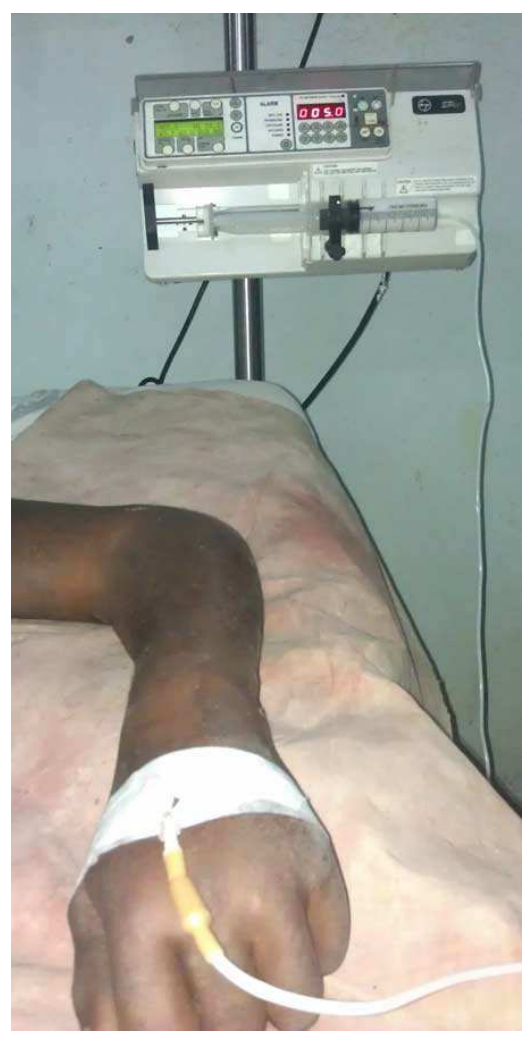

